\title{
REVIEW
}

\section{Treatment of Systemic Juvenile Idiopathic Arthritis with Tocilizumab - the Role of Anti-Interleukin-6 Therapy After a Decade of Treatment}

\author{
Andreas Reiff
}

To view enhanced content go to www.biologicstherapy-open.com Received: December 8, 2011 / Published online: February 1, 2012

(C) The Author(s) 2012. This article is published with open access at Springerlink.com

\section{ABSTRACT}

With the recent approval of tocilizumab as the first biologic for the treatment of systemic juvenile idiopathic arthritis (sJIA), an important unmet medical need for this historically challenging disease has now been met. The purpose of this review article is to revisit the established therapeutic options for sJIA, to summarize the history of the clinical trials with tocilizumab, and to discuss its role in the treatment of sJIA.

Keywords: anti-interleukin-6; systemic juvenile idiopathic arthritis; tocilizumab

\section{A. Reiff $(\bowtie)$}

Division of Rheumatology, Childrens Hospital Los Angeles, Los Angeles, CA, USA.

Email: areiff@chla.usc.edu

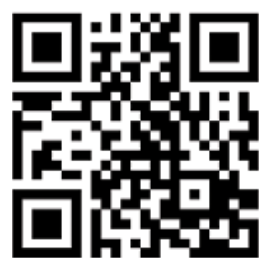

Enhanced content for Biologics in Therapy articles is available on the journal web site: www.biologicstherapy-open.com

\section{INTRODUCTION}

Systemic juvenile idiopathic arthritis (sJIA) affects between $5 \%$ and $15 \%$ of all children with JIA in North America and Europe [1]. In Asia, however (including India and Japan), this form of the disease may account for up to $50 \%$ of all JIA cases $[2,3]$. Despite being relatively rare, sJIA is responsible for nearly two thirds of all childhood arthritis mortality [4-6]. Although sJIA is equally distributed among girls and boys and can occur at any age, it often affects younger children $[7,8]$.

The diagnosis of sJIA is a diagnosis of exclusion requiring a thorough work-up of infectious diseases and malignancies, especially in those patients in whom arthritis is absent at onset. Diagnostic criteria include a history of arthritis in any number of joints and at least 2 weeks of daily monophasic or biphasic fevers accompanied by at least one of the following:

- evanescent, salmon-pink-colored rash on the trunk or overlying the joints;

- hepatosplenomegaly;

- generalized lymphadenopathy;

- $\quad$ serositis (pericarditis, pleuritis).

It is well known that arthritis may be absent at disease onset, which often leads to delays 
in early diagnosis. Characteristic lab findings include leukocytosis, anemia of chronic inflammation, thrombocytosis, elevated acutephase reactants, and signs of disseminated intravascular coagulation [9].

Macrophage activation syndrome (MAS), especially during the active systemic phase of the disease, represents the most serious complication and is associated with a high mortality rate $[10,11]$.

Prior to the introduction of the newer biologic treatments approximately one third of sJIA patients had a monophasic disease course and more than half developed erosive polyarticular arthritis, with potential long-term disability $[2,9,12]$.

Although the etiology of sJIA remains unknown, recent scientific evidence seems to suggest that this subform of JIA has many traits of an autoinflammatory rather than an autoimmune disorder, and leukocyte transcriptional signatures can be used to distinguish sJIA from other febrile illnesses [13]. Pascual et al. analyzed transcriptional patterns of freshly isolated blood mononuclear cells from patients with active sJIA in addition to analyzing transcriptional changes induced upon culturing healthy blood mononuclear cells with serum from active sJIA patients [14]. The results revealed that sJIA serum upregulated interleukin-1 (IL-1) transcription in healthy cells and that IL-1 protein secretion was induced in a disease-activity-dependent manner. IL-1 $\beta$ induced genes such as pentraxin 3, potassium inward-rectifier channel, subfamily J, member 15 (KCNJ15), or adenosine triphosphate (ATP)sensitive inward rectifier potassium channel were found to be upregulated in peripheral blood mononuclear cells in the majority of active sJIA patients. These patterns normalized after treatment with anakinra. Additionally, mononuclear cells from patients with active sJIA were found to secrete excess IL-1 $1 \beta$ protein upon activation, while interleukin-6 (IL-6) and tumor necrosis factor (TNF) production did not differ significantly from controls, suggesting a specific dysregulation of the IL-1 pathway in patients with SJIA [14].

As reviewed previously, IL-1 is the key cytokine in autoinflammatory syndromes and an important mediator of sJIA $[15,16]$. IL-1 $\beta$ and IL-1 are formed from pro-IL-1 via caspase activity. While IL-1 is mostly membrane-bound, IL- $\beta$ is synthesized by monocytes, macrophages, and dendritic cells and plays a pivotal role in the induction of fever and rash and stimulates the level of circulating IL-6 [12-15]. In addition, other cytokines such as IL-6, interleukin-7 (IL-7), and interleukin-18 (IL-18) play a more dominant role in this disease than in other forms of JIA. Increased plasma levels of IL- 6 and IL- 1 result in higher levels of acutephase reactants and myeloid-related proteins (MRP 8 and 14), increased production and mobilization of platelet and white blood cells, and anemia [15-23].

Conversely, IL-6 is a pleiotropic cytokine that regulates immune responses and inflammatory reactions. IL-6, alone or with other cytokines, interacts with various cells such as T cells, B cells, lymphocytes, monocytes, and fibroblasts, driving synovial proliferation, inflammation, autoimmunity, and destruction of articular structures through the activation of osteoclasts [24]. Both membrane-bound interleukin-6 receptor (IL-6R) and the soluble form of IL-6R are able to mediate proinflammatory IL-6 signaling through the interaction with the cytokine receptor subunit glycoprotein gp130 [25]. Like IL-1, IL-6 is associated with many of the manifestations of sJIA such as fever, rash, lymphadenopathy, hepatosplenomegaly, anemia, and poor growth [21,22]. Markedly increased IL-6 levels in serum and synovial fluid 
are one of the hallmark features of sJIA. IL-6 is especially elevated during or just before fever spikes, while it is often only marginally elevated in between fever attacks [18].

\section{METHODS}

An extensive literature search was conducted using the PubMed and Ovid medical databases. Keywords included search terms such as "juvenile idiopathic arthritis," "systemic," "arthritis," "macrophage activation syndrome," "treatment," "anakinra," "IL-1ra," "rilonacept," and "tocilizumab," and searches for interleukins 1, 6, 15, and 18.

\section{TREATMENT FOR sJIA}

Traditionally treatment for sJIA has been challenging, partly as a result of long-term corticosteroid toxicity and limited efficacy of traditional disease-modifying antirheumatic drugs (DMARDs) such as methotrexate and cyclosporine $[26,27]$. On the other hand, due to the autoinflammatory nature of sJIA, anti-TNF agents have shown very limited efficacy in sJIA in comparison to other forms of JIA [28-30].

Subsequent to the recognition that IL-1 dysregulation may contribute to the pathogenesis of sJIA [31], multiple studies have demonstrated the dramatic clinical impact of IL-1 blockade in the form of either an IL-1 receptor antagonist (IL-ra, anakinra), a monoclonal anti-IL-1 antibody (canakinumab), or rilonacept (an IL-1 type I receptor and IL-1 receptor accessory protein fusion protein also known as IL-1 Trap), with rapid induction of clinical remission in patients with sJIA [32-39].

A recently published study [32] retrospectively examined anakinra as first-line therapy (either in monotherapy [10/46 patients] or in combination with steroids and or DMARDs [36/46 patients] in 46 children with active sJIA (all with active fevers and rash) with a median age of 7.5 years who were started on treatment an average of 80 days after disease onset. Treatment was associated with rapid resolution of systemic symptoms and, more remarkably, prevention of arthritis in almost $90 \%$ of patients during the observation period. The authors concluded that treatment with anakinra was most favorable when initiated early in the disease, before arthritis had become well established. In most cases, treatment with anakinra avoided protracted corticosteroid use and prevented the development of severe, therapy-resistant arthritis.

A similar observation was made by Gattorno et al. in their study of 22 patients with active systemic-onset JIA (fever in $17 / 22$ and rash in 12/22) [33]. They found that complete responders to anakinra had a smaller number of joints with active disease at baseline and a higher absolute neutrophil count compared with patients who were incomplete responders or nonresponders.

In another study, by Pascual et al., anakinra treatment in 9 children with active sJIA (7/9 with fevers and rashes) resulted in a complete treatment response in 7/9 [31]. The patients with the best response to anakinra had less articular involvement and a higher total white blood cell count, similar to those observed in the Gattorno study.

Lastly, in the study by Quartier et al. in which 24 children with sJIA were treated with anakinra (mean age 8.5 years, mean disease duration 3.7 years, and mean active joint count 16), the initial beneficial treatment effect in most patients was lost over time, illustrating that a treatment response to IL-1 inhibition is less favorable in those patients with more advanced sJIA, since only about a third of patients in this study had active fevers at baseline [34].

The rilonacept trial enrolled 24 patients, all with fevers and or rash. The median age was 14 years, the mean disease duration was 3 years, 
and the average joint count was 10 . Patients received rilonacept $2.2 \mathrm{mg} / \mathrm{kg}$ or $4.4 \mathrm{mg} / \mathrm{kg}$ or placebo subcutaneously, with a maximum allowable weekly dose of $320 \mathrm{mg}(4.0 \mathrm{~mL})$. A history of anakinra use was recorded in $29 \%$ of the patients. At 3 months from study initiation, the adapted American College of Rheumatology (ACR) pediatric 30, 50, and 70 responder rates were $78.3 \%, 60.9 \%$, and $34.8 \%$, respectively, which improved further during the open-label period. In patients previously treated with anakinra $(n=7)$, the adapted ACR pediatric 70 responder rate at the 12 -month evaluation was $85.7 \%(6 / 7)$. The clinically significant reduction in systemic disease manifestations that was observed during the double-blind phase was maintained during open-label treatment, which exemplifies again the beneficial role of anti-IL-1 treatment in the early stages of sJIA $[38,39]$.

Finally, similar observations were made in the various treatment studies with canakinumab. In the initial phase 2 dose escalation study of canakinumab in 25 patients with advanced sJIA (median disease duration of 38 months and a mean of 20 [range, 4-62] swollen joints at baseline), patients received canakinumab in doses ranging from 0.5 to $9 \mathrm{mg} / \mathrm{kg}$. Seventeen of them had been previously treated with anakinra. Fifty-nine percent of the children reached an ACR pediatric 50 response within 15 days, and 4 patients achieved inactive disease. Six of the eleven nonresponders to anakinra responded to a single dose of canakinumab. Notably, nonresponders had a substantially higher number of active joints (median, 33.5 joints) and somewhat lower levels of systemic inflammation relative to responders (median, 9 joints) [36].

Comparable results were seen in the subsequent two phase 3 studies with canakinumab in 84 patients with sJIA [40]. In this study the average age of patients was 8.5 years and the average disease duration was fairly short at 2 years. All patients had fevers and/or rash, but the inclusion criteria required those symptoms to be present only for at least a day. Even though the average swollen joint count was about 8 , the range was fairly high, with some patients having up to 58 swollen joints. After a month of treatment, ACR pediatric 50 and 100 were achieved by $76 \%$ and $32 \%$ of the patients compared to only $5 \%$ and $2.4 \%$ of the patients on placebo. In general, monthly dosing with canakinumab (4 mg/kg/month) significantly reduced the risk of flare and enabled successful steroid tapering/discontinuation while demonstrating an acceptable safety and tolerability profile. Infections, predominantly of the upper respiratory tract, some serious, were the most frequent adverse events reported. A total of 5 cases of MAS were observed including 4 patients in the canakinumab and one patient in the placebo groups, three of which were considered life-threatening, and two of which resulted in death [40].

\section{Safety of Anti-IL-1 Treatments}

To my knowledge, none of the published anti-IL-1 trials reported treatment-related deaths, malignancies, or opportunistic infections, including tuberculosis. In general infections occur more frequently with anti-IL-1 agents than in placebo-treated patients, and serious infections have been reported but appear to be generally rare.

\section{Treatment with Tocilizumab}

Tocilizumab (Actemra ${ }^{\circledR}$; Hoffmann-La Roche Ltd., Ontario, Canada) is a humanized, monoclonal anti-IL-6 receptor antibody that binds to soluble and membrane-bound IL-6 receptors, specifically blocking IL-6 actions [24]. 


\section{History}

Two phase 2 clinical trials and one phase 3 trial demonstrating the clinical efficacy and safety of tocilizumab in systemic-onset JIA have been published [41-43].

Like the clinical trials with anti-IL-1 agents, most trials employed the modified ACR pediatric definition of improvement for assessment in sJIA, which addresses the improvement of the systemic inflammatory features of the disease [44]. Fever and characteristic rash are key clinical features of SJIA, and the modified ACR pediatric criteria require meeting the ACR definition of improvement and improvement or resolution of sJIA-associated fever and rash [45].

The first clinical trial examining the evidence for tocilizumab in the treatment of sJIA was published by Yokota et al. after initial compassionate use of tocilizumab in four children with sJIA [41]. In this doubleblind intention-to-treat trial with open-label run-in, successive randomization, and openlabel extension, 56 Japanese children (aged 2-19 years) with treatment-refractory sJIA were given three doses of tocilizumab $8 \mathrm{mg} / \mathrm{kg}$ every 2 weeks during a 6-week open-label lead-in phase. At the end of the open-label lead-in phase, ACR pediatric 30,50 , and 70 responses were achieved by 51 (91\%), 48 (86\%), and 38 (68\%) of the patients, respectively. Forty-three patients continued to the double-blind phase, where $4 / 23$ patients $(17 \%)$ in the placebo group maintained an ACR pediatric 30 response and a C-reactive protein (CRP) concentration of less than $15 \mathrm{mg} / \mathrm{L}$ compared with 16 of $20(80 \%)$ in the tocilizumab group $(P<0.0001)$. By week 48 of the open-label extension phase, 47 (98\%), 45 (94\%), and 43 (90\%) of 48 patients, respectively, achieved ACR pediatric 30, 50, and 70 responses [43]. The most common adverse events included mild infections and transient increases in transaminases. Serious adverse events included anaphylactoid reactions, gastrointestinal hemorrhage, bronchitis, and gastroenteritis.

This trial was followed by an open-label phase 2 trial of single, ascending doses of tocilizumab in 18 caucasian children with sJIA in the UK and France [42]. All patients had evidence of active advanced sJIA based on a mean joint count of 21 swollen joints despite treatment with $>0.2 \mathrm{mg} / \mathrm{kg} /$ day of prednisolone and methotrexate $\left(\leq 20 \mathrm{mg} / \mathrm{m}^{2} /\right.$ week) in 12 patients. Patients received a single dose of 2,4 , or $8 \mathrm{mg} / \mathrm{kg}$ of tocilizumab by intravenous (i.v.) infusion. Treatment responses were rapid and continued even after serum tocilizumab levels became undetectable. Clinical improvement in these children was observed for up to 8 weeks, with 11/18 patients achieving ACR pediatric 30 and $8 / 18$ patients ACR pediatric 50 or better. There were no observable differences with age and no evidence of dose-limiting toxicity. One of the major shortcomings of this trial is the fact that no adapted ACR score specific for sJIA was used and the authors did not comment specifically on the impact of tocilizumab on the systemic features of the disease.

These two trials eventually paved the way for a phase 3 randomized double-blind placebocontrolled trial with tocilizumab in patients with active sJIA (the TENDER trial) [46]. The first part of this 5-year ongoing multinational study examined the safety and efficacy of tocilizumab administered i.v. for 12 weeks in 112 children with active sJIA. The primary endpoint in this portion of the study was the proportion of subjects achieving at least an ACR pediatric 30 response plus absence of fever at week 12.

Children aged 2-17 (average age 9.5) with an average disease duration of 5 years were randomized to receive placebo or tocilizumab at two different doses based on body weight. The doses used in this study were based on 
pharmacokinetic modeling in children in the two earlier studies, and differed from the typical adult rheumatoid arthritis dose. Tocilizumab was given at $12 \mathrm{mg} / \mathrm{kg}$ for children with a body weight $<30 \mathrm{~kg}$ or $8 \mathrm{mg} / \mathrm{kg}$ for children with a body weight $\geq 30 \mathrm{~kg}$ i.v. every 2 weeks. Patients were randomly assigned 2:1 to receive tocilizumab or placebo i.v. every 2 weeks for 12 weeks in a double-blind manner. Prior to enrollment, study participants had to have had active sJIA for at least 6 months and be unable to tolerate or not have responded adequately to previous treatments. In addition, patients had to have either five or more active joints or two or more active joints plus fever higher than $38^{\circ} \mathrm{C}$ for 5 or more days during the 14-day screening period.

Patients were allowed to continue on stable doses of methotrexate, nonsteroidal antiinflammatories, or corticosteroids at a maximum dose of $0.5 \mathrm{mg} / \mathrm{kg} /$ day. In a significant fraction of the study patients prior anti-TNF and anti-IL-1 therapy had failed. The mean age of the patients was 9 years, the average disease duration was 5 years, and the average joint count at baseline was 20 . However, only a little more than half of the patients $(65 / 112$ or $58 \%)$ had fevers and only 40/112 (36\%) had rashes [46].

\section{Efficacy}

At 12 weeks, $85 \%$ of participants receiving tocilizumab achieved the primary endpoint ( $\geq 30 \%$ improvement in ACR pediatric 30 and absence of fever) compared with $24 \%$ of children receiving placebo. As a secondary endpoint $71 \%$ and $37 \%$ of the children in the tocilizumab group achieved ACR pediatric 70 and 90 responses by week 12 , compared to only $8 \%$ and $5 \%$ respectively of children who received placebo.

In the second part of the trial, which examined long-term safety and efficacy, 48\% of the patients had no actively swollen joints,
$80 \%$ achieved an ACR pediatric 70 response, and 59\% achieved an ACR pediatric 90 response at week 52 of the open-label extension [47]. In addition, the mean oral corticosteroid dose decreased from 0.3 to $0.06 \mathrm{mg} / \mathrm{kg}$, with discontinuation in more than half of the patients. Significant differences were observed between tocilizumab and placebo for all levels of response. Tocilizumab-treated patients were 31.5 -times $(P<0.0001)$ more likely to achieve the primary outcome. Treatment outcomes at week 52 were independent of prior methotrexate use and were similar in patients who had received biologic treatment prior to baseline compared to those with no prior biologic treatment. However, a higher proportion of patients who had not received prior biologic treatment achieved an ACR pediatric 90 response. This outcome was maintained after 2 years, when $88 \%$ of the remaining 65 patients maintained an adapted ACR pediatric 70 and $46 \%$ an adapted ACR pediatric 90 response. Fifty-five percent of the patients no longer had actively swollen joints and about a third of the patients were considered to be in remission [47].

\section{Safety}

The most frequent side effects in the study were upper respiratory infections, headache, nasopharyngitis, and diarrhea. The most commonly reported infections were pneumonia, gastroenteritis, chickenpox, and ear infections; these were more frequently seen in tocilizumabtreated patients. During the double-blind phase, the frequency of adverse events was similar in the two groups, with four serious adverse events in tocilizumab-treated patients. After 2 years, there had been 47 serious adverse events in 35 patients, including 22 serious infections, leading to a death from sepsis in one patient [47]. Of note, since tocilizumab blocks the production of CRP as an acute-phase reactant, monitoring CRP 
as marker for infection (or disease activity) may not be useful. Despite the absence of evidence of anti-tocilizumab antibodies at baseline, infusion reactions were reported in $16 \%$ of patients in the tocilizumab group and 5\% of patients in the placebo group, including two separate anaphylactic reactions in one tocilizumab-treated patient, who eventually was withdrawn from the study. Two patients developed anti-tocilizumab antibodies during treatment and were withdrawn due to adverse events.

Three MAS episodes were reported, one considered by the investigator as possibly related to tocilizumab. In the other two patients withdrawal of tocilizumab and incomplete dosing might have contributed to MAS development. All patients recovered with standard treatment.

Data from the 1-year analysis demonstrated grade 3 neutropenia $\left(0.5-1.0 \times 10^{9} / \mathrm{L}\right)$ occurring in 17 patients and grade 4 neutropenia $\left(<0.5 \times 10^{9} / \mathrm{L}\right)$ in 2 . All episodes resolved without sequelae and were not associated with serious infection.

Liver enzyme elevations (alanine transaminase $[\mathrm{ALT}])$ to grade 2 or worse $(\geq 2.5$ times upper limit of normal [ULN]) occurred in 21 patients, and at least one value of total cholesterol and low density lipoprotein (LDL) cholesterol above the ULN was recorded in 31\% and $14 \%$ of patients, respectively, compared with only one patient in the placebo group.

Opportunistic infections and tuberculosis were not reported. A total of three deaths occurred during the long-term observation period of the trial: one in a 17-year-old boy from tension pneumothorax at week 50 who had a been well controlled on the study drug (ACR pediatric 90 response), one in a 4 -year-old boy from suspected streptococcal sepsis possibly related to tocilizumab, and one in a 10 -year-old boy involved in road traffic accident unrelated to tocilizumab. In addition to these three deaths, three additional deaths occurred in children who had completed the study and were no longer receiving tocilizumab. The first was in a 13-year-old boy with a 2-year history of sJIA who died 6 months after the last dose of tocilizumab. Immediately before death he developed severe pulmonary hypertension and neurological issues. The exact cause of death was listed as unknown and the investigator assessed the death as related to a complication of the sJIA and as unrelated to tocilizumab. The second patient was almost 18 years old with a nearly 13-year history of sJIA at the time of death. She had very severe treatment-resistant sJIA and had been treated with three DMARDS and four biologics prior to enrollment in the study. She was hospitalized for cardiac failure and was found to have pulmonary veno-occlusive disease, pulmonary hypertension, and congestive heart failure. She died acutely 54 weeks after her last tocilizumab dose from an atrioventricular block secondary to hyperkalemia. The investigator assessed the pulmonary veno-occlusive disease as unrelated to tocilizumab. The third and final patient was almost 19 years old at the time of death and had had sJIA for slightly over 7 years. He initially had an excellent response to tocilizumab (ACR pediatric 90) but was withdrawn from treatment due to a flare with recurrence of fever and arthritis. Over a year later, he died suddenly and the primary cause of death was reported as cardiac arrest. The investigator considered this event as unrelated to the study medication.

\section{DISCUSSION}

\section{Where does Tocilizumab Fit in the Treatment of sJIA?}

The approval of tocilizumab as the first biologic for the treatment of sJIA has enriched our treatment armamentarium for this historically 
challenging disease. Similar to findings of previous trials with anti-TNF agents in polyarticular JIA, clinical outcomes with tocilizumab have demonstrated rapid onset and dramatic clinical improvements that have been sustained during the long-term observation periods. In addition, unlike canakinumab, tocilizumab may decrease the occurrence of MAS, which significantly influences the mortality risk in sJIA patients $[40,46,47]$. However, while the safety profile of the drug appears to be acceptable overall, the occurrence of five deaths (a sixth death was unrelated) in patients exposed to tocilizumab in the TENDER trial should heighten our awareness with regard to future safety data. Tocilizumab should be avoided in children with active acute or chronic infections, a known history of elevated liver or lipid levels, and neutropenia. Special caution should be used in children with a history of serious infusion reactions to other biologic agents.

While the results of the TENDER trial are very encouraging, the study population can hardly be described as a "systemic" population since only a little more than half of the patients $(65 / 112$, or $58 \%$ ) had fevers and only 40/112 (36\%) had rashes at baseline. In addition, the average disease duration was 5 years and the average joint count was 19 [46].

Based on a growing body of literature about the treatment of sJIA, it becomes increasingly clear that SJIA requires a more refined, diseasestage-specific treatment strategy. Unfortunately, clinical trials in sJIA suffer from the fact that some patients with sJIA have a monocyclic disease course with spontaneous remission and frequently exclude patients in the early phase of the disease. In addition, patients in this early phase often have not yet been seen by the pediatric rheumatologist during the work-up for infection or malignancy. For the clinician, however, this group of patients is often the most

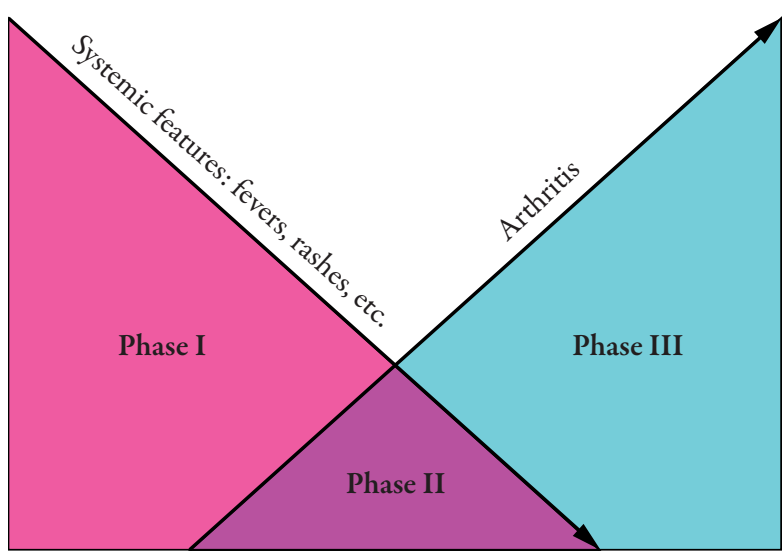

Interleukin-1

Interleukin-6

Tumor necrosis factor

Fig. 1. Course of systemic juvenile idiopathic arthritis (sJIA). Although part of a continuous spectrum and variable between patients, sJIA seems to follow a certain cytokine predominance that defines the various disease stages.

challenging in terms of diagnosis, treatment, and risk of MAS.

Based on the summary of the clinical evidence from the various studies above, it appears that sJIA follows a fairly distinct cytokine pattern that correlates well with the in-vitro immunologic studies and the clinical response to the individual anticytokine treatments.

Therefore, the author would like to propose that sJIA is one disease entity with three fairly distinct but overlapping phases along the course of disease progression, recognizing that not all patients may go through all of these phases (Fig. 1). This differentiation is based on evidence from the published literature including treatment responses to various biologics as reviewed above and my personal clinical experience. Patients may regress to prior phases due to intermittent infections or recurrent disease flares.

\section{Phase 1}

The active systemic phase is characterized by the presence of systemic disease manifestations 
including fevers, rashes, hepatosplenomegaly, laboratory evidence of macrophage activation with elevated acute phase reactants (CRP, fibrinogen, thrombocytosis, anemia of chronic inflammation, elevated d-dimers, etc.), and disseminated coagulation. Initially fevers and rashes may not yet follow a classic quotidian or bi-quotidian pattern. In the past this disease was often referred to as Wissler syndrome, since patients often only had systemic symptoms including arthralgias but mostly no evidence of true arthritis [48]. This phase may precede the development of arthritis by weeks or even, in rare cases, years [9].

Clinically this phase most closely resembles an autoinflammatory syndrome, and anti-IL-1 therapy appears to be the most effective treatment. Preliminary evidence seems to suggest that anti-IL-1 therapy in this phase might even prevent further disease progression [32]. In the personal opinion of the author, the role of anti-IL- 6 therapy in this phase remains unclear, while treatment with anti-TNF agents appears to have little or no effect.

\section{Phase 2}

In this overlap phase, arthritis is becoming increasingly prevalent, while systemic symptoms are still present but tend to be less prominent and regular than in phase 1 . The literature quotes a progression to aggressive, erosive polyarticular arthritis in up to $80 \%$ of sJIA patients [2,3]. In this phase treatment with anti-IL-1 therapy appears to be less effective than in phase 1 . Studies with rilonacept and canakinumab suggest a beneficial response in $50 \%-60 \%$ of the patients (see above). At the same time treatment with anti-TNF agents remains suboptimal. In this phase, treatment with anti-IL- 6 agents such as tocilizumab appears to be the best treatment strategy.

\section{Phase 3}

As patients progress further in their disease course, arthritis is becoming the predominant symptom, while systemic symptoms are less prevalent or absent. In this phase treatment with tocilizumab appears still to be very effective. However, anti-TNF treatment may regain some efficacy. Evidence about a treatment response to anti-TNF therapy in this phase can be found in the early etanercept and adalimumab trials where patients with sJIA were allowed to enroll as long as they had no active systemic symptoms [49-51]. Interestingly, some of the sJIA patients in the etanercept trial experienced systemic flare during the placebo phase in the double-blind randomized portion and could not be recaptured with etanercept once they were allowed to restart the active drug (unpublished personal observation). Treatment with anti-IL-1 therapy in this phase is minimally effective, as reported in the initial trial of anakinra in 80 children with a polyarticular course of JIA and similar to the outcomes seen in rheumatoid arthritis [37].

\section{CONCLUSION}

sJIA is a prime example of a rare childhood disease in which innovative approaches in basic science and trial and error in clinical trials with various modern biologic agents have taught us valuable lessons about the pathophysiology. It is apparent that sJIA requires disease-stagespecific treatment that will ultimately lead to better outcomes and a decrease in mortality.

\section{ACKNOWLEDGMENTS}

Dr Reiff is a speaker and consultant for Amgen, Abbott, Genentech, Novartis, and Pfizer. He has been an investigator for products from the above-mentioned companies. 
Dr Reiff is the guarantor for this article, and takes responsibility for the integrity of the work as a whole.

Open Access. This article is distributed under the terms of the Creative Commons Attribution Noncommercial License which permits any noncommercial use, distribution, and reproduction in any medium, provided the original author(s) and source are credited.

\section{REFERENCES}

1. Cassidy JT, Petty RE, Laxer RM, Lindsley CB. Chronic arthritis in childhood. In: Textbook of Pediatric Rheumatology. 6th edition. Philadelphia: Saunders; 2011:211.

2. Lomater C, Gerloni V, Gattinara M, Mazzotti J, Cimaz R, Fantini F. Systemic onset juvenile idiopathic arthritis: a retrospective study of 80 consecutive patients followed for 10 years. J Rheumatol. 2000;27:491-496.

3. Wallace CA, Levinson JE. Juvenile rheumatoid arthritis: outcome and treatment for the 1990's. Rheum Dis Clin North Am. 1991;17:891-905.

4. Ansell BM, Wood PN. Prognosis in juvenile chronic polyarthritis. Clin Rheumatic Dis. 1976;2:397-412.

5. Fujikawa S, Okuni M. A nationwide surveillance study of rheumatic diseases among Japanese children. Acta Paediatr Jpn. 1997;39:242-244.

6. Seth V, Kabra SK, Semwal OP, et al: Clinicoimmunological profile in juvenile rheumatoid arthritis - an Indian experience. Indian J Pediatr. 1996;63:293-300.

7. Ogilvie EM, Fife MS, Thompson SD, et al. The $-174 \mathrm{G}$ allele of the interleukin-6 gene confers susceptibility to systemic arthritis in children: a multicenter study using simplex and multiplex juvenile idiopathic arthritis families. Arthritis Rheum. 2003;48:3202-3206.

8. Sullivan DB, Cassidy JT, Petty RE. Pathogenic implications of age of onset in juvenile rheumatoid arthritis. Arthritis Rheum. 1975;18:251-255.

9. Cassidy JT, Petty RE, Laxer RM Lindsley CB. Systemic juvenile idiopathic arthritis. In: Textbook of Pediatric Rheumatology. 6th edition. Saunders 2011:241.

10. Davì $\mathrm{S}$, Consolaro $\mathrm{A}$, Guseinova $\mathrm{D}$, et al. MAS Study Group. An international consensus survey of diagnostic criteria for macrophage activation syndrome in systemic juvenile idiopathic arthritis. J Rheumatol. 2011;38:764-768.

11. Ravelli A. Macrophage activation syndrome. Curr Opin Rheumatol. 2002;14:548-552.

12. Singh-Grewal D, Schneider R, Bayer N, Feldman BM. Predictors of disease course and remission in systemic juvenile idiopathic arthritis: significance of early clinical and laboratory features. Arthritis Rheum. 2006;54:1595-1601.

13. Mellins ED, Macaubas C, Grom AA. Pathogenesis of systemic juvenile idiopathic arthritis: some answers, more questions. Nat Rev Rheumatol. 2011;7:416-426

14. Pascual V, Allantaz F, Patel P, Palucka AK, Chaussabel D, Bancherau J. How the study of children with rheumatic diseases identified interferon-alpha and interleukin-1 as novel therapeutic targets. J Immunol Rev. 2008;223:39-59.

15. Reiff A. The use of anakinra in juvenile arthritis. Curr Rheumatol Rep. 2005;7:434-440.

16. Dinarello CA. Blocking IL-1 in systemic inflammation. J Exp Med. 2005;201:1355-1359.

17. De Benedetti F, Martini A. Is systemic juvenile rheumatoid arthritis an interleukin 6 mediated disease? J Rheumatol. 1998;25:203-207.

18. De Benedetti F, Massa M, Robbioni P, Ravelli A, Burgio GR, Martini A. Correlation of serum interleukin-6 levels with joint involvement and thrombocytosis in systemic juvenile rheumatoid arthritis. Arthritis Rheum. 1991;34:1158-1163.

19. De Benedetti F, Pignatti P, Gerloni V, et al. Differences in synovial fluid cytokine levels between juvenile and adult rheumatoid arthritis. J Rheumatol. 1997;24:1403-1409.

20. De Benedetti F, Alonzi T, Moretta A, et al. Interleukin 6 causes growth impairment in transgenic mice through a decrease in insulin-like growth factor-I. A model for stunted growth in children with chronic inflammation. J Clin Invest. 1997;99:643-650.

21. De Benedetti F, Rucci N, Del Fattore A, et al. Impaired skeletal development in interleukin-6- 
transgenic mice: a model for the impact of chronic inflammation on the growing skeletal system. Arthritis Rheum. 2006;54:3551-3563.

22. Cazzola M, Ponchio L, de Benedetti F, et al. Defective iron supply for erythropoiesis and adequate endogenous erythropoietin production in the anemia associated with systemic-onset juvenile chronic arthritis. Blood. 1996;87:48244830 .

23. Frosch M, Ahlmann M, Vogl T, et al. The myeloidrelated proteins 8 and 14 complex, a novel ligand of toll-like receptor 4 , and interleukin-1beta form a positive feedback mechanism in systemic-onset juvenile idiopathic arthritis. Arthritis Rheum. 2009;60:883-891.

24. Mihara M, Kasutani K, Okazaki $M$, et al. Tocilizumab inhibits signal transduction mediated by both mIL-6R and sIL-6R, but not by the receptors of other members of IL- 6 cytokine family. Int Immunopharmacol. 2005;5:1731-1740.

25. Jones SA, Richards PJ, Scheller J, Rose-John S. IL-6 transsignaling: the in vivo consequences. J Interferon Cytokine Res. 2005;25:241-253.

26. Woo P. Systemic juvenile idiopathic arthritis: diagnosis, management, and outcome. Nat Clin Pract Rheumatol. 2006;2:28-34.

27. Hashkes PJ, Laxer RM. Medical treatment of juvenile idiopathic arthritis. JAMA. 2005;294:1671-1684.

28. Quartier P, Taupin P, Bourdeaut F, et al. Efficacy of etanercept for the treatment of juvenile idiopathic arthritis according to the onset type. Arthritis Rheum. 2003;48:1093-1101.

29. Horneff G, Schmeling $H$, Biedermann $T$, et al. The German etanercept registry for treatment of juvenile idiopathic arthritis. Ann Rheum Dis. 2004;63:1638-1644.

30. Kimura Y, Pinho P, Walco G, et al. Etanercept treatment in patients with refractory systemic onset juvenile rheumatoid arthritis. J Rheumatol. 2005;32:935-942.

31. Pascual V, Allantaz F, Arce E, Punaro M, Banchereau J. Role of interleukin-1 (IL-1) in the pathogenesis of systemic onset juvenile idiopathic arthritis and clinical response to IL-1 blockade. J Exp Med. 2005;201:1479-1486.

32. Nigrovic PA, Mannion M, Prince FH, et al. Anakinra as first-line disease-modifying therapy in systemic juvenile idiopathic arthritis: report of forty-six patients from an international multicenter series. Arthritis Rheum. 2011;63:545-555.

33. Gattorno $M$, Piccini $A$, Lasigliè $D$, et al. The pattern of response to anti-interleukin-1 treatment distinguishes two subsets of patients with systemiconset juvenile idiopathic arthritis. Arthritis Rheum. 2008;58:1505-1515.

34. Quartier P, Allantaz F, Cimaz R, et al. A multicentre, randomised, double-blind, placebo-controlled trial with the interleukin-1 receptor antagonist anakinra in patients with systemic-onset juvenile idiopathic arthritis (ANAJIS trial). Ann Rheum Dis. 2011;70:747-754.

35. Zeft A, Hollister R, LaFleur B, et al. Anakinra for systemic juvenile arthritis: the Rocky Mountain experience. J Clin Rheumatol. 2009;15:161-164.

36. Ruperto N, Quartier P, Wulffraat N, for the Paediatric Rheumatology International Clinical Trials Organisation (PRINTO). A phase II study to evaluate dosing and preliminary safety and efficacy of canakinumab in systemic juvenile idiopathic arthritis with active systemic features. Arthritis Rheum. 2011. [Epub ahead of print].

37. Ilowite $\mathrm{N}$, Porras $\mathrm{O}$, Reiff $\mathrm{A}$, et al. Anakinra in the treatment of polyarticular-course juvenile rheumatoid arthritis: safety and preliminary efficacy results of a randomized multicenter study. Clin Rheumatol. 2009;28:129-137.

38. Lovell D, Giannini E, Kimura Y, et al. Preliminary evidence for sustained bioactivity of IL-1 trap (Rilonacept), a long acting IL-1 inhibitor, In: Systemic Juvenile Idiopathic Arthritis (SJIA). Arthritis Rheum. 2007;56(suppl. 9):Abstract 1282.

39. Lovell DJ, Giannini EH, Kimura Y, et al. Longterm safety and efficacy of rilonacept in patients with systemic juvenile idiopathic arthritis (SJIA). Arthritis Rheum. 2009;60(suppl. 10):Abstract 2053.

40. Brunner H, Ruperto N, Horneff G, et al. Phase III study results on the efficacy and safety of canakinumab, a long acting fully human interleukin $-1 \beta$ antibody in systemic idiopathic juvenile arthritis with active systemic features. Arthritis Rheum. 2011;63(suppl. 10):Abstract 2622.

41. Yokota S, Miyamae T, Imagawa $\mathrm{T}$, Katakura S, Kurosawa R, Mori M. Clinical study of tocilizumab in children with systemic-onset juvenile idiopathic arthritis. Clin Rev Allergy Immunol. 2005;28:231-238.

42. Woo P, Wilkinson N, Prieur AM, et al. Open label phase II trial of single, ascending doses of MRA 
in Caucasian children with severe systemic juvenile idiopathic arthritis: proof of principle of the efficacy of IL- 6 receptor blockade in this type of arthritis and demonstration of prolonged clinical improvement. Arthritis Res Ther. 2005; 7:R1281-1288

43. Yokota S, Imagawa T, Mori M, et al. Efficacy and safety of tocilizumab in patients with systemiconset juvenile idiopathic arthritis: a randomised, double-blind, placebo-controlled, withdrawal phase III trial. Lancet. 2008;371:998-1006.

44. Giannini EH, Ruperto N, Ravelli A, Lovell DJ, Felson DT, Martini A. Preliminary definition of improvement in juvenile arthritis. Arthritis Rheum. 1997;40:1202-1209.

45. Ramanan A, Schneider R, Batthish $\mathrm{M}$, et al. Developing a disease activity tool for systemiconset juvenile idiopathic arthritis by international consensus using the Delphi approach. Rheumatology. 2005;44:1574-1578.

46. De Benedetti F, Brunner $\mathrm{H}$, Allen $\mathrm{R}$, et al. Tocilizumab Is efficacious in patients with systemic juvenile idiopathic arthritis across baseline demographic and disease characteristics and prior/baseline treatments: 52-week data from a phase 3 clinical trial. Arthritis Rheum. 2011;63(suppl. 10):Abstract 2621.

47. De Benedetti F, Brunner $\mathrm{H}$, Ruperto $\mathrm{N}$, et al. Efficacy and safety of tocilizumab in patients with systemic juvenile idiopathic arthritis: 2 year data from a phase III clinical trial. Arthritis Rheum. 2011;63(suppl.):L111.

48. Wissler H. Subsepsis allergica [in German]. Ergeb Inn Med Kinderheilkd. 1965;23:202-220.

49. Lovell DJ, Giannini EH, Reiff A, et al. Etanercept in children with polyarticular juvenile rheumatoid arthritis. Pediatric Rheumatology Collaborative Study Group. N Engl J Med. 2000;342:763-769.

50. Reiff AO, Lovell DJ, Ilowite NT, et al. Safety and efficacy of over 8 years of continuous etanercept $\left(\right.$ Enbrel $\left.^{\circledR}\right)$ therapy in patients with juvenile rheumatoid arthritis. Arthritis Rheum. 2008;58:1496-1504.

51. Lovell DJ, Ruperto N, Goodman S, et al. Pediatric Rheumatology Collaborative Study Group; Pediatric Rheumatology International Trials Organization. Adalimumab with or without methotrexate in juvenile rheumatoid arthritis. N Engl J Med. 2008;359:810-820. 